\title{
The new method of electroencephalogram representation
}

doi:10.1080/11762320701455658

\author{
R. T. Al-kasasbeh ${ }^{1}$ and R. A. Shepovalnikov ${ }^{2}$ \\ ${ }^{l}$ Al-Balqa'Applied University/Faculty of Engineering Technology, Electrical Engineering Department, \\ Amman, Fordan \\ ${ }^{2}$ St. Petersburg State Electrotechnical University (LETI), Russia
}

\begin{abstract}
A new method for visualization of electroencephalogram process is developed, using factorial analysis. This method permits data compression and visualization in a form adequate for humans to trace continuous change patterns, compare data from brain hemispheres and delete apparent artifacts using combined man-computer mode for achieving additional reliability. Accordingly, this method is realized in software that allows an operator to select various modes of representation of results and speed of visualization.
\end{abstract}

Key words: electroencephalogram, factorial analysis, automation, visualization, dynamic representation, man-machine system, reliability.

\section{INTRODUCTION}

The analysis of time-continuous relations of fluctuations in biopotential concurrently in many points of a brain provides a great volume of the computing operations connected to the correlation or coherent analysis of electroencephalogram signals. This next led to development of both hardware and software systems for research automation. An effective methodical example for similarity analysis of electroencephalogram signals appeared representation of correlation matrix EEG in three-dimensional factorial space (Tsitseroshin 1986; Tsitseroshin et al. 2000). However, static representation of results of factorial analysis EEG limits opportunities of the analysis and ordering of the observable dynamic phenomena accompanying because of fast reorganization of structure of a biopotential field of a brain which by itself suggests dynamic representation.

In the following work we develop an appropriate program and adequate means for visualization of results of factorial analysis EEG which allow the researcher to visually trace dynamics of system bioelectric processes of a brain in real time and in convenient forms, thus partially solving the problem.

Corresponding Author:

R. T. Al-Kasasbeh

Al-Balqa'Applied University

Nour Al Houda, Abu Nsear, Building 57

Ammam-11937, PO Box 541324 Jordan

Tel: +962777787972

Email: rjordanjo@yahoo.co.uk

\section{DEVELOPMENT}

First we provide the basic theoretical preconditions and concepts of multivariate statistics which are used in particular, in the analysis of multiple channel EEG signals. An effective way of a compression of the information contained in correlation matrixes EEG, it is a factorial analysis (FA) of the initial data (Blagush 1989; Ajvazjan et al. 1989).

The other methods of EEG representations are network architecture for classifying feature vectors symbolizing portions of an electroencephalogram (EEG) trace of a human subject (Felzer and Freisleben 2003) and the spectral decomposition of source EEG signals (Burger and Hoppe 2007; Akin and Kiymik 2000). Evidently, that the visual analysis of positional relationship of dozens vectors in multivariate space is to the utmost inconvenient. These vectors, if statistically dependent, can be approximately transformed into space of some dimension $\mathrm{m}$, with $m$ possibly considerably less than number of parameters $n$. Coordinate axles of such reduced space are some general factors $F_{m}$ and initial parameters $\times_{i}$ can be linearly expressed through them:

$$
\begin{gathered}
\times_{i}=a_{i 1} F_{1}+a_{i 2} F_{2}+\cdots+a_{i m} F_{m}+d_{i} U_{i} \\
\quad(i=1,2,3, \ldots, n) .
\end{gathered}
$$

It is the basic model of the classical FA. Here $\times_{i}$ and $F_{m}$ are random variables, as well as the characteristic factor $U_{i}$. The classical model of the factorial analysis, in our case, gives the following form:

$$
\begin{aligned}
\times_{1}(t)= & a_{11} F_{1}(t)+a_{12} F_{2}(t)+\cdots+a_{1 j} F_{j}(t)+\cdots \\
& +a_{1 m} F_{m}(t)+d_{1} U_{1}(t)
\end{aligned}
$$




$$
\begin{aligned}
& \mathrm{\times}_{2}(t)=a_{21} F_{1}(t)+a_{22} F_{2}(t)+\cdots+a_{2 j} F_{j}(t)+\cdots \\
& +a_{2 m} F_{m}(t)+d_{2} U_{2}(t) \\
& \times_{i}(t)=a_{i 1} F_{1}(t)+a_{i 2} F_{2}(t)+\cdots+a_{i j} F_{j}(t)+\cdots \\
& +a_{i m} F_{m}(t)+d_{i} U_{i}(t) \\
& \times_{n}(t)=a_{n 1} F_{1}(t)+a_{n 2} F_{2}(t)+\cdots+a_{n j} F_{j}(t) \ldots \\
& +a_{n m} F_{m}(t)+d_{n} U_{n}(t) \text {. } \\
& (m \ll n)
\end{aligned}
$$

Here $n$ of EEG processes are linearly connected with $m$, the common for all constituent factors $F_{j}(t)$ which in this model represent mutually orthogonal processes. The number $m$ provided by adequately chosen algorithm of FA is usually significantly less then number $n$ of outputs of EEG. Each of the EEG processes also depends linearly on one local, not interconnected statistically with other processes, the so-called characteristic factor $U_{j}(t)$. Its value determines the contribution of local component EEG in the given lead.

During EEG representation in separate brains on the CRT screen by radiuses - vectors as projections on the plane from multivariate factorial space - it was found that angles between vectors are inversely proportional to correlation between corresponding EEG leads (Tsitseroshin and Pogosjan 1993). Thus, the closer the vectors in factorial space EEG, the larger is the above correlation between these processes. And inversely, at angles approaching to 90 degrees, correlation coefficients are close to zero.

In consecutive periods of EEG analysis (duration of each of 4 seconds), after their transformation to the digital form, degree of statistical affinity of signals between each pair of the leads $(1-2,1-3,1-4 \ldots 1-n, 2-3,2-4,2-5 \ldots 2-$ $n, 3-4,3-5,3-6, \ldots n-n)$ is calculated. On the basis of these calculations correlation matrices are created. After FA EEG is carried out by centroid method, the weight of first three factors contains usually more than $80 \%$ of the information about structure of a correlation matrix (Blagush 1989; Ajvazjan et al 1989).

As a result, FA values of three factors for all EEG leads (from 12 up to 20) and consecutive periods of analysis EEG turn out. The received data can be displayed on the screen as radiuses-vectors and are used for the visual analysis. However, such an approach does not provide an opportunity of animation that can be essentially important, if necessary investigate dynamics of process. For the solution of this problem it is expedient to keep results of FA in a databank from where one can take them in a sequence and then it will be possible to take, compare and analyze them.

EEG is most commonly recorded according to the international 10-20 electrode placement system shown in Figure 1 .

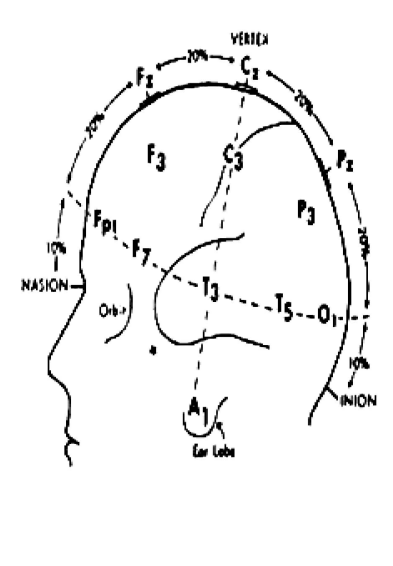

LEFT SIOE

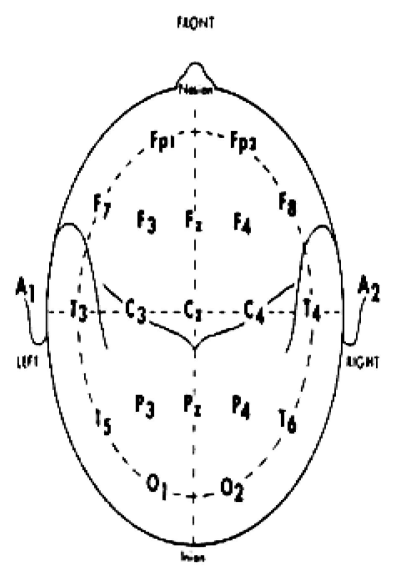

TOP
Figure 1 Distribution of electrodes.

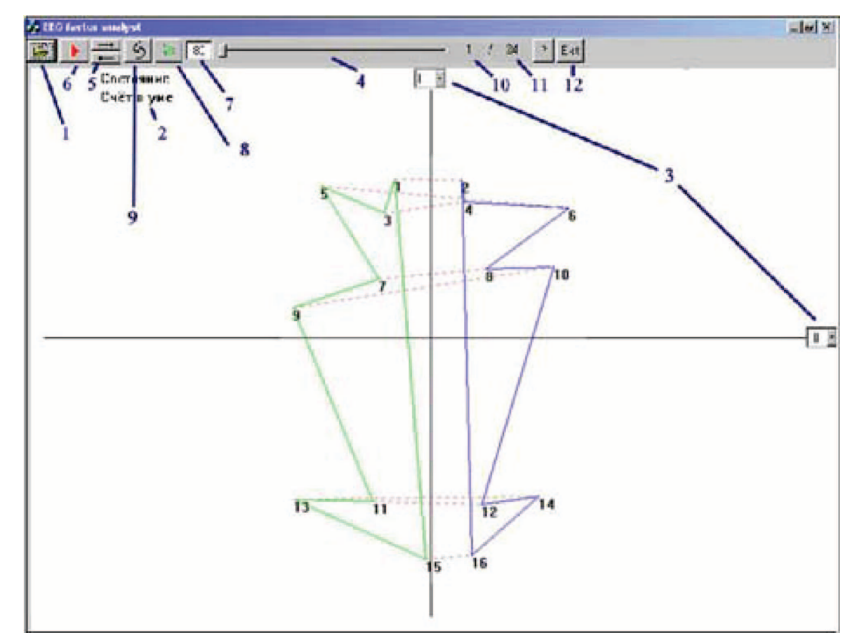

Figure 2 The graphical user interface of the program for EEG representation.

The 10-20 system (Bogacz et al. 1999) was developed to standardize the collection of EEG data and facilitate the comparison of studies performed at different laboratories. When only a few channels of EEG are collected the electrodes are placed at a subset of the sites. The recorded signal is obtained by subtracting a signal measured below the eye from one measured above the eye.

On further data processing (for example, for studying dynamics of factors values changes) it is possible to use specialized software developed by us. It is written in Microsoft Visual Studio 6.0 (Swart 2005; Holzner 2000). The graphical user interface of developed software is represented in the Figure 2.

The program allows the operator to choose for research: a plane of factors, a period of the analysis, a dynamic or static mode of display, various modes of representation of results and speed of visualization.

Let's review in detail an algorithm of the program which allows us to carry out the following operations in particular: 
1. Display of factorial analysis EEG results in static (traditional) and dynamic mode of displaying. Depending on one's desire, it is possible to use various modes of display.

For realization of animation the value of factors on an interval between the next period for both displayed factors and for all leads are calculated by consecutive approach of value of the factor from last by the subsequent period of the analysis. These values of factors on all periods of the analysis are known from a file with the data of FA.

In the analysis of the data it is convenient to use the accelerated viewing, as it is possible to find out easily and quickly sharp changes, in particular biological and technical artifacts which frequently deform the EEGSIGNAL.

2. Display of the processes occurring in bilateral symmetric areas of the left and right hemisphere of a brain.

Display lead number I in factorial space is designated by one of figures 1-16 which corresponds to the number of an electrode - for the left hemisphere - even for the right odd figures (Figures 3(a) and (b)).

With a traditional mode of display the center of coordinates of factorial space incorporates a display of each lead in factorial space. Thus the color of lines of the connection going to display of electrodes located on left hemisphere, becomes green, and color of the lines going to display of electrodes located on right hemisphere becomes blue. At a mode offered by us the display of leads located above different hemispheres is united in factorial space in two groups. Displays of electrodes of the left hemisphere incorporate the order of increase 1-16, display of last electrode 16 (that corresponds to occipital lead) incorporates to display 1 (frontal lead). The similar picture is under construction for the right hemisphere.

In a case when on axes are chosen II-d and III-rd factors additional shaped lines are drawn: they connect displays of symmetric electrodes located in different hemispheres.

3. Viewing autocorrelation function. Value of the factor on an axis of $\mathrm{x}$-coordinate remains same, as well as at traditional representation of results FA EEG and value on an axis of ordinates will correspond to value of the factor for a period of the analysis earlier. Autocorrelation function allows us to fix occurrence of changes of $\mathrm{FA}$ results effectively.

4. Display of the contribution of value of each factor in color of the image of brains contours. The structure of a biopotential field of a brain can be submitted as a color ellipse (corresponding to a brain contour) in which color of pixel is calculated under the normal law depending on size of each of three factors and distance from pixel to display of corresponding lead EEG. Thus red color corresponds to the first factor, green color to the second, blue color to the third. A combination of factors (i.e., different combinations of intensities of red, green and blue colors) determines color of pixel.

Thus, the algorithm developed by us allows one to carry out dynamic display of results of the factorial analysis of the

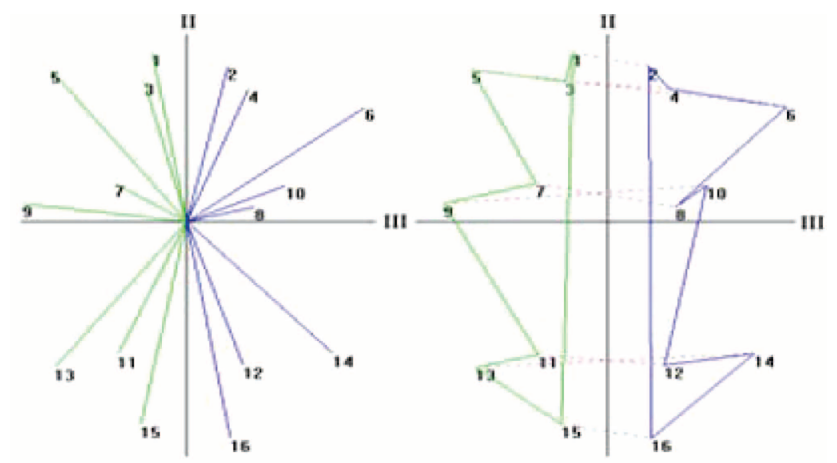

Figure 3 Representation of results FA EEG in a plane formed by axes 2 nd and 3 rd factors for 16 leads. (a) traditional form of representation, (b) the mode offered by us.

\section{Table 1 The result of using FA software}

\begin{tabular}{llll}
\hline & & \multicolumn{2}{c}{ Result of analysis } \\
\cline { 3 - 4 } Diseases & $\begin{array}{l}\text { Number of } \\
\text { Realization }\end{array}$ & $\begin{array}{l}\text { Coincidence } \\
\text { with expert }(\%)\end{array}$ & $\begin{array}{l}\text { False alarm } \\
(\%)\end{array}$ \\
\hline Schizophrenia & 36 & 89.1 & 10.9 \\
Brain stress & 33 & 91 & 9 \\
Brain damage & 29 & 90.2 & 9.8 \\
\hline
\end{tabular}

fluctuations of brain bioelectric activity. For the researcher most of the information is provided by changes in mutual disposition of vectors, by changes in weights of orthogonal $1,2,3$ factors and also by appearance or strengthening of $4,5,6$ factors.

\section{RESULTS}

For practical implementation of the developed method we chose three types of diseases: schizophrenia, brain stress and brain damage. For classification of diseases EEGs of 98 patients were used. The result of the classification using specialized software of factorial analysis (FA) developed by us is represented in Table 1:

The results represented in Table 1 show that the proposed method of factorial analysis much more sensitive in the field of schizophrenia and brain stress diagnosis as compare with traditional methods. As we can see, measurement of 98 patients' records shows approximately a $90 \%$ of successful diagnosis. Also, very favorable results were acquired by applications of the algorithm to diagnostics of brain stress and brain damage in new born children.

The diagnosis significance of the suggested method is illustrated in Figure 4.

Top panel, projections of electroencephalography radial vectors on the plane of factors I-II; bottom panel, on the plane of factors II-III. Numbers of EEG radial vectors (112), which correspond to EEG leads as shown on the left. A healthy person in a state of rest has a steady distribution of EEG radial vectors, which reproduce the location of corresponding EEG leads in abstract three-dimensional 


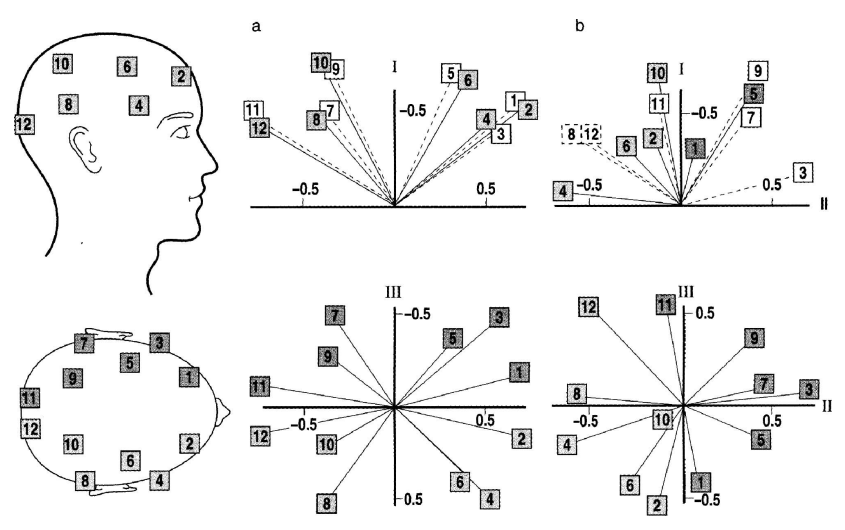

Figure 4 The EEG vectors representation of brain activity in normal (a) and pathological (b) states.

space. In case of pathology (e.g., a patient with concussion of the brain) this order is disrupted.

\section{DISCUSSION}

It can be easily seen, that this algorithm is to be especially effective when there is not local, but system damage to the brain. Algorithm provides essential advantages in comparison to the traditional way of static representation of results, as additional opportunities to use potential advantages of system "man-machine" are created. Due to mobility of the image the researcher can estimate a condition of the examinee not only on displays of leads in factorial space, but also on the dynamics of their moving. As the spatial arrangement of radius-vectors with a high degree of affinity reflects an arrangement of electrodes in a surface of a head, the opportunity to estimate dynamics of brain processes is created, and it is of paramount value for studying laws of neurological-and-mental activity.

The accelerated animation provides more effective search of rough changes EEG, that is, allocation of artifacts that allows to approach to the decision important, actual and still far from the decision the problem of automatic exclusion of artifacts on EEG.

Autocorrelation function is expedient for using for revealing those electrodes, the potential under which changes to the greatest degree and as when change of values only one of factors takes place.

Usage of the mode of display of results FA for division of electrodes of different hemispheres allows us to present more evidently functional interaction of symmetric departments of the left and right hemisphere (for example, 1-2, $3-4)$. Especially it is appreciable, when on factorial axes are chosen IInd and IIIrd factors as in this mode additional shaped lines which connect displays of symmetric electrodes are drawn. Parallelism of these straight lines concerning an axis of IInd factor is shown as far as similar to the organization of processes in a right and left hemisphere.

\section{CONCLUSION}

It is necessary to note that the described program can be used not only for analysis EEG, but also for the analysis of any other types of three factorial data for which the average size of values of factors differs less than on the order.

Here we should mention that the formidable task of finding and deleting artifacts in EEG and similar biomedical data is now proceeding with attempts to use different methods. There are, for example, attempts to use neural network systems for finding and deleting artifacts (Tsitseroshin and Pogosjan 1993; Swart 2005; Holzner 2000). Gibson and James (Clochon et al. 1992) use independent constraind analysis for seizure onset analysis of EEG. Al-Kasasbeh, Al-Kasasbeh and Lvov (Bogacz et al. 1999; Gibson and James in press; Jasper 1958) also used factor analysis wavelet and fractal analysis technologies for finding artifacts. However, as one could have expected, these approaches are in the experiment phase and do not provide general solutions.

We expect that visualization ideas from our software can be different from FA for such methods. Also combined methods can be effective.

\section{REFERENCES}

Ajvazjan SA, Buhshtaber VM, Enjukov IS, et al. 1989. Applied statistics: Classification and decrease of dimension: Finance and statistics, pp. 607.

Akin M, Kiymik M. 2000. Application of periodogram and AR spectral analysis to EEG signals. $\mathcal{F}$ Med Syst 24(4):247-256.

Al-Kasasbeh RT. 2004. Wavelet-based method for EEG artifacts classification. IFMBE Proceedings, Kuala Lumpur, Malaysia, 2-4.09, pp. 157-159.

Al-Kasasbeh RT, Lvov BV. 2005. Classification of EEG signals with artifacts based on fractal dimension analysis, wavelet transform and neural network. Dirasat International Journal, Vol. 32, pp. 78-90. Jordan.

Al-Kasasbeh RT. 2006. Detection of eye movement and muscle artifacts in EEG of normal subjects by classification of fractal dimension dynamics. Dirasat International Journal, Vol. 33. Jordan.

Al-Kasasbeh RT. 2007. Two dimensional representation of spacial structure changes in brain bioelectric potential field. Presented at 3rd Kuala Lumpur International Conference in Biomechanical Engineering. Berlin: Springer.

Blagush P. 1989. Factor analysis with features: The finance and statistics, pp. 248.

Bogacz R, Markowska-Kaczma U, Kozlik A. 1999 Blinking artifact recognition in EEG signal using neural network. Proc 4th conference neural networks and Applications. Poland Zakopane, p. 6.

Burger M, Hoppe U. 2007. Wavelet-based analysis of MMN. Biomed Tech (Berl). 52(1):111-6.

Clochon P, Perchey G, Couque C, et al. 1992. Automatized classification by neural networks of EEG signals with artefact rejection. Proc 14th Ann Int Conf IEEE Eng Med and BiolSoc, Lyon, France: Satellite Symosium on Neuroscience and Technology, pp. 51-55. 
Durka P, Kiezyk R, Blinowska K. 1996. Neural networks and wavelet analysis in EG artifact recognition.

II Konferencia Siec Neuronowe CH Zastosowanie szczyrk $301 \mathrm{~V} 4$ p. 6 (htpp://brain.fuw.edu.pi/ durka/pd_paper. html

Felzer T, Freisleben B. 2003. Analyzing EEG signals using the probability estimating guarded neural classifier. IEEE Trans Neural Syst Rehabil Eng, 11(4):361-71.

Gibson O, James C. 2002. Constraind ICA for seizure onset analysis in the EEG. Biomedical Information Engineering Research Group, Aston University, Birmingham, UK.

Holzner Z. 2000. Visual C ++6 : a training course. SPb: Peter, $576 \mathrm{pp}$.
Jasper HH. 1958. The ten-twenty electrode system of the international federation. Electroencephalogr Clin Neurophysiol, 10:371(373).

Swart Bob. 2005. Borland ${ }^{\circledR}$ C ++ Builder ${ }^{\circledR}$ Migration to.NET using VCL for NET ISBN 1-57268-059-4.

Tsitseroshin MN. 1986. Analysis of statistical interrelation of fluctuations of brain biopotentials in three-dimensional factorial space. Autometria, 6:89.

Tsitseroshin MN, Pogosjan AA. 1993. About display of activity integrative mechanisms of a brain and its bioelectric activity. Biophysics, 38(2): 341.

Tsitseroshin MN, Pogosjan AA, Galperina EI. et al. 2000. System interaction cortical fields at realization verbal - memory activity. Human Physiol, 26(6): 20-30. 

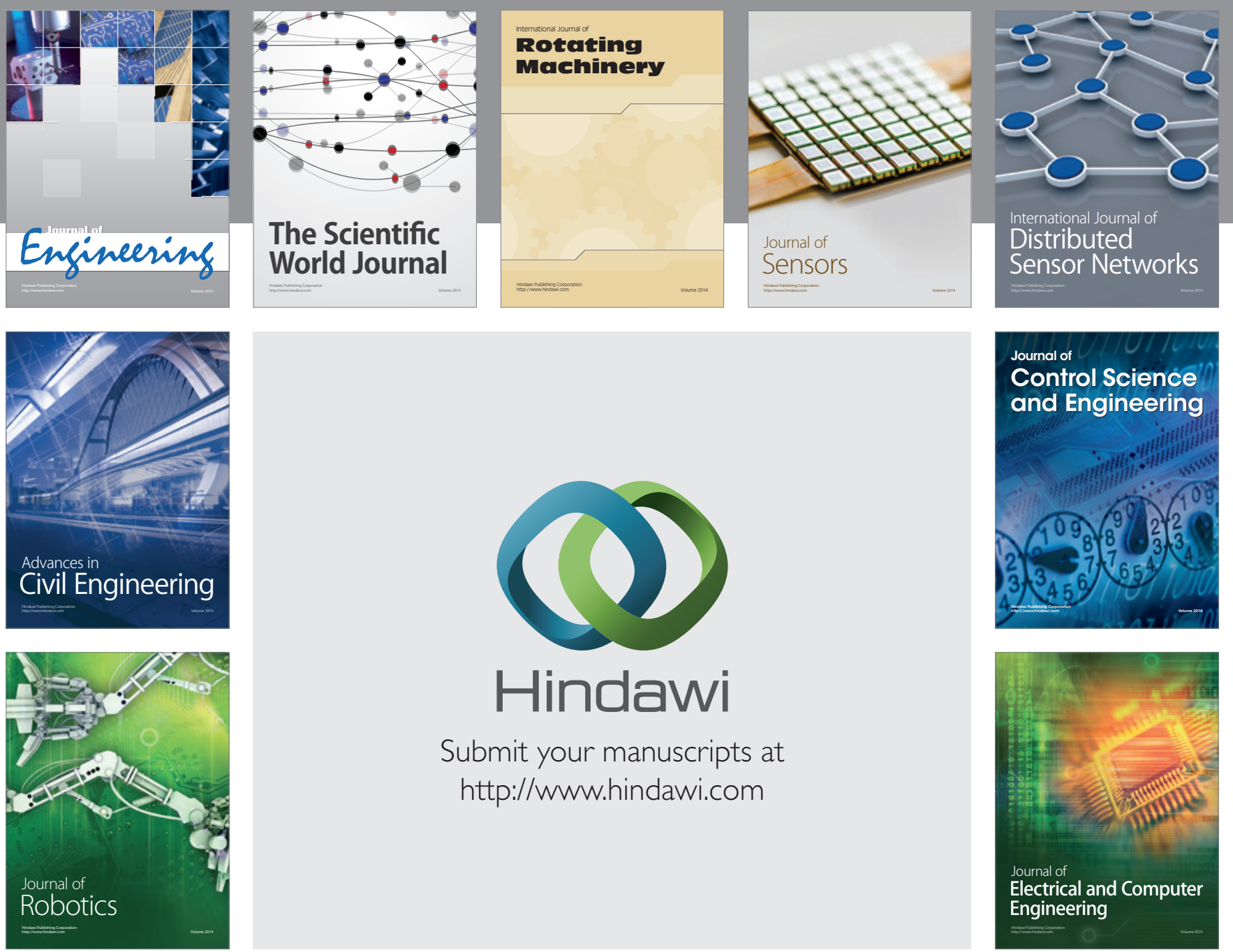

Submit your manuscripts at

http://www.hindawi.com
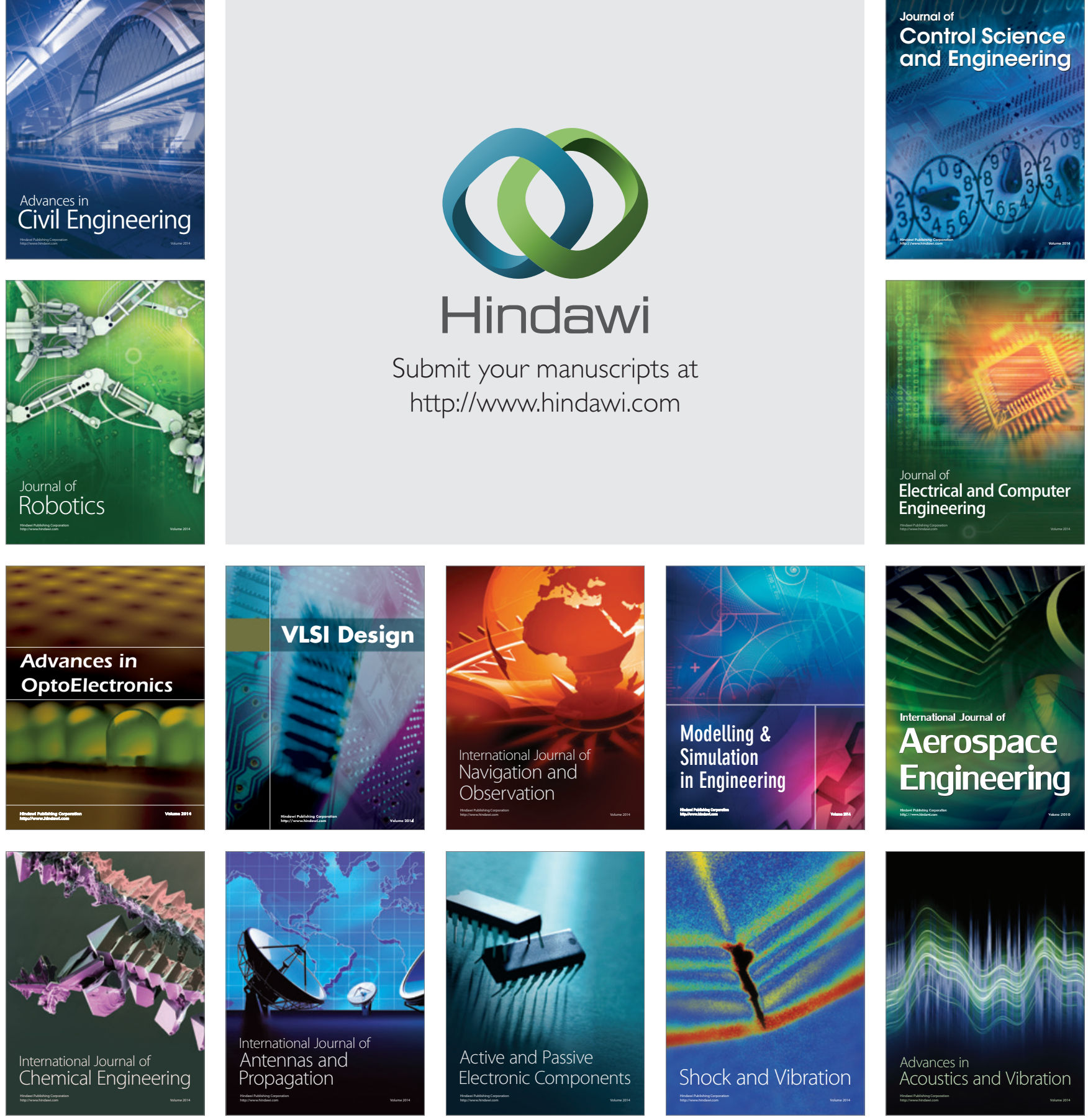